\title{
QUANTIZATION OF SYMPLECTIC COBORDISMS
}

\author{
Levente Korpás and Alejandro Uribe \\ Dedicated to Victor Guillemin on his 60th birthday
}

\begin{abstract}
In this paper we define the notion of quantized cobordism as a unitary operator between $\operatorname{Spin}^{c}$ quantizations of cobordant symplectic manifolds, and announce a theorem from [13] describing the microlocal structure of this operator.
\end{abstract}

\section{Introduction}

Ginzburg, Guillemin and Karshon have used the concept of symplectic cobordism to provide elegant new proofs of some theorems in symplectic geometry (the Duistermaat-Heckman theorem and the Jeffrey-Kirwan localization theorem), see [10]. The point is that the natural cohomological invariants of symplectic manifolds are cobordism-invariant while the relation of symplectic cobordism is relatively weak, so that "complicated" symplectic manifolds are cobordant to "simple" ones. In particular the Riemann-Roch polynomial is a cobordism invariant. On the other hand, it has been observed that it is possible to quantize integral compact symplectic manifolds by the $\mathrm{Spin}^{c}$ Dirac operator, in a way that yields a Hilbert space with dimension equal to the Riemann-Roch number of the manifold (at least if one allows oneself to multiply the symplectic form by a large integer, see [4]). One is led to wonder what the relationship is between this quantization and the relation of symplectic cobordism at an analytical level, with a view for example to using the cobordism relation to simplify the quantization of complicated objects. This is the subject of the present paper.

We will use the following definition of symplectic cobordism.

Definition 1.1. Let $\left(M_{1}, \omega_{1}\right)$ and $\left(M_{2}, \omega_{2}\right)$ be two compact symplectic manifolds of dimension $2 n$ (not necessarily connected). A symplectic cobordism from $M_{1}$ to $M_{2}$ is an oriented $2 n+1$ dimensional manifold $X$, with boundary, endowed with a closed 2 -form, $\sigma$, such that:

(1) $\sigma$ is non-degenerate, and so its kernel is a rank-one subbundle $\mathcal{V} \subset T X$;

(2) $\partial X=M_{1} \amalg M_{2}^{-}$as oriented manifolds, where $\partial X$ has the boundary orientation, $M_{1}$ the orientation induced by $\omega_{1}^{n}=\omega_{1} \wedge \cdots \wedge \omega_{1}$ and $M_{2}^{-}$ the orientation induced by $-\omega_{2}^{n}$;

(3) for $j=1,2$, the pull-back of $\sigma$ to $M_{j}$ under the inclusion $M_{j} \hookrightarrow X$ equals $\omega_{j}$.

Received November 30, 1998. Revised January 22, 1999.

Second author supported in part by NSF grant DMS-9623054. 
The symplectic cobordism is called integral if the form $\sigma$ represents an integral cohomology class in $H^{2}(X, \mathbb{R})$. In this case there exists a "prequantum" line bundle, i.e. a complex line bundle $\mathcal{L} \rightarrow X$ with connection $\nabla^{\mathcal{L}}$ such that $\operatorname{curv}\left(\nabla^{\mathcal{L}}\right)=-i \sigma$.

The bundle $\mathcal{V}$ is oriented by the following condition: A section $\nu$ of $\mathcal{V}$ is positive if there exists locally $2 n$ vector fields $\xi_{1}, \ldots \xi_{2 n}$ such that $\sigma^{n}\left(\xi_{1}, \ldots \xi_{2 n}\right)>0$ and $\left(\nu, \xi_{1}, \ldots \xi_{2 n}\right)$ is positive with respect to the given orientation of $M$. Therefore $\mathcal{V}$ is trivial, and we will denote by $\nu$ a positive non-vanishing section of $\mathcal{V}$. Since the pull-back of $\sigma$ to the boundary is the symplectic form, $\nu$ is transverse to the boundary. Clearly, $\nu$ restricted to $M_{1}$ (resp. $M_{2}$ ) is inward-pointing (resp. outward-pointing).

An oriented odd-dimensional manifold $X$ equipped with a non-degenerate closed two-form $\sigma$ is called an extended phase space by Arnold, ([1], Chapter 9), and "espace d'évolution" by J.M. Souriau, ([18], Chapter 3), based on the following particular case: If $(M, \omega)$ is a symplectic manifold and $H \in C^{\infty}(M)$, then one can set:

$$
X=[0, T] \times M \quad \text { and } \quad \sigma=\omega+d t \wedge d H .
$$

It is well-known that in this case the integral curves of $\nu$ can be naturally identified with the integral curves of the Hamilton flow of $H$ on $M$ (for times $0 \leq t \leq T)$. A symplectic cobordism can be viewed as a generalization of this example where one does not have a well-defined notion of time, and the non-trivial topology of $X$ allows for the possibility that the characteristic relation

(1.2) $\Gamma:=\left\{(x, y) \in M_{1} \times M_{2} \mid x\right.$ and $y$ are on the same trajectory of $\left.\nu\right\}$

is not the graph of a smooth canonical transformation $M_{1} \rightarrow M_{2}$. Indeed, not all integral curves of $\nu$ that start at $M_{1}$ reach $M_{2}$ so the domain of $\Gamma$ is not all of $M_{2}$. (We will denote the image, resp. the domain of $\Gamma$ by $M_{1}^{\circ}$ and $M_{2}^{\circ}$.) $\Gamma$ is, however, an open Lagrangian submanifold of $M_{1} \times M_{2}^{-}$which we think of as the graph of a symplectic surgery, based on some of the examples we present in $\S 4$. Accordingly, we will call $M_{j} \backslash M_{j}^{\circ}$ the surgery set.

Motivated by Example (1.1) (and some K-theoretic considerations that we won't go into), our thesis here is that the quantization of a symplectic cobordism from $M_{1}$ to $M_{2}$ ought to yield a unitary operator from the quantization of $M_{1}$ to that of $M_{2}$, which in the semi-classical limit corresponds to the canonical relation $\Gamma$. Our approach however is not to try to generalize the fundamental solution of the Schrödinger equation, since the latter does not have the covariance properties required by our setting. Instead, since we are quantizing the manifolds $M_{j}$ by $\operatorname{Spin}^{c}$, we will seek an operator that realizes the cobordism invariance of the index.

In $\S 2$ we define the quantization of an integral symplectic cobordism. Our definition of the quantization of $(X, \sigma)$ follows L. Nicolaescu's proof of the 
cobordism-invariance of the index, [15]. In $\S 3$ we announce some of the results of [13] on the semi-classical properties of this quantization, and in $\S 4$ we take a brief look at some low-dimensional examples.

\section{The definition}

For the rest of the paper we let let $(X, \sigma)$ be an integral symplectic cobordism. The quantization of $(X, \sigma)$ depends on the choice of a Riemannian metric, $\langle\cdot, \cdot\rangle$, which is compatible with the form $\sigma$, in the following sense: Let $\mathcal{W} \subset T X$ denote the metric normal of the line bundle spanned by the vector field $\nu$. Then $\mathcal{W}$ is a symplectic vector bundle and compatibility means that the skew symmetric bundle endomorphism, $J$, defined on $\mathcal{W}$ by

$$
\langle v, w\rangle_{x}=\sigma_{x}(v, J w) \quad \text { for all } x \in X \text { and } v, w \in \mathcal{W}_{x}
$$

is an almost complex structure. We also require that in some collar neighborhood, $(-1,0] \times \partial X$, of the boundary the metric is a product metric. (This condition can probably be weakened to an asymptotic statement, i.e. to a $b$ metric in the sense of Melrose, without changing the main results.) It is easy to see that compatible metrics always exist.

$\operatorname{Spin}^{c}$ Dirac operators. Given a compatible metric and associated almostcomplex structure on $\mathcal{W}$, we can canonically construct a bundle $\mathcal{S}_{\mathbb{C}} \rightarrow X$ of irreducible complex Clifford modules for $\mathbb{C} \ell\left(T^{*} X\right)$. We let

$$
\mathcal{S}_{\mathbb{C}}:=\bigwedge\left(\mathcal{W}^{0,1}\right)^{*}
$$

and define the Clifford action of $w \in \mathcal{W}^{*} \subset T^{*} M$ on a section $\varphi \in C^{\infty}\left(\mathcal{S}_{\mathbb{C}}\right)$ as follows. First decompose $w$ as $w^{1,0}+w^{0,1}$ according to $\mathcal{W}^{*} \subset \mathcal{W}^{*} \otimes \mathbb{C}=$ $\left(\mathcal{W}^{1,0}\right)^{*} \oplus\left(\mathcal{W}^{0,1}\right)^{*}$, then set

$$
c(w) \cdot \varphi=\sqrt{2}\left(w^{0,1} \wedge \varphi-i\left(w^{1,0}\right) \varphi\right),
$$

where $i\left(w^{1,0}\right)$ denotes contraction with $w^{1,0}$, using the $\mathbb{C}$-linear extension of the metric to $\mathcal{W}^{*} \otimes \mathbb{C}$. We assume that $\nu$ is normalized and let $\eta$ be its metric dual. To define the action of $\eta$, let $\left\{e^{1}, e^{2}, \ldots, e^{2 n}\right\}$ be a local orthonormal frame of $\mathcal{W}^{*}$ and set

$$
c(\eta):=\varepsilon i^{n-1} c^{1} c^{2} \cdots c^{2 n}, \quad \text { where } \quad c^{j} \equiv c\left(e^{j}\right) .
$$

Choosing +1 or -1 for $\varepsilon$, we obtain two bundles of Clifford modules $\mathcal{S}_{\mathbb{C}}^{+}$and $\mathcal{S}_{\mathbb{C}}^{-}$ whose fiber at any point $x \in X$ realizes the two inequivalent irreducible complex representations of $\mathbb{C} \ell\left(T_{x}^{*} X\right)$. Note that in the decomposition

$$
\mathcal{S}_{\mathbb{C}}^{+}=\bigwedge^{\text {even }}\left(\mathcal{W}^{0,1}\right)^{*} \oplus \bigwedge^{\text {odd }}\left(\mathcal{W}^{0,1}\right)^{*},
$$

the operator $c^{0}:=c(\eta)$ acts as $-i I d^{\text {even }}+i I d^{\text {odd }}$. The chirality operator

$$
\chi=i^{n+1} c^{0} c^{1} \cdots c^{2 n}
$$


acts on the Clifford modules $\mathcal{S}_{\mathbb{C}}^{+}$and $\mathcal{S}_{\mathbb{C}}^{-}$as +1 and -1 respectively. We will set $F:=\mathcal{S}_{\mathbb{C}}^{+}$. (For related discussion see [3], pp. 109-110.)

As usual, to define a $\operatorname{Spin}^{c}$ Dirac operator, $D$, on sections of $F \rightarrow X$ we fix a connection $\nabla$ on $F$ compatible with the above Clifford action, and set (locally) $D=\sum_{i=0}^{2 n} c\left(e^{i}\right) \nabla_{\xi_{i}}$, where $\left\{\xi_{0}, \ldots, \xi_{2 n}\right\}$ is a local frame of $T X$ and $\left\{e^{0}, \ldots, e^{2 n}\right\}$ is the corresponding dual frame.

Notice that if we write the Dirac operator on $F \rightarrow X$ in the form

$$
D=c(\eta)\left(\nabla_{\nu}+\tilde{A}\right)
$$

then $\tilde{A}$ is a totally characteristic operator on $X$ whose boundary value is the $\operatorname{Spin}^{c}$ Dirac operator [9], $A$, on the bundle $E:=\left.F\right|_{\partial X}$. (More precisely the canonical $\operatorname{Spin}^{c}$ Dirac operator of the boundary is $c(\eta) A$, where $c(\eta)$ is a bundle automorphism.) We will denote by $L \rightarrow \partial X$ the restriction of the line bundle $\mathcal{L} \rightarrow X$ to the boundary and consider the twisted Dirac operators:

$$
D^{k} \text { on } F \otimes \mathcal{L}^{k}, \quad A_{j}^{k} \text { on } E_{j} \otimes L_{j}^{k},
$$

where we have denoted by $L_{j} \rightarrow M_{j}$ (resp. $E_{j} \rightarrow M_{j}$ ) the restriction of $L$ to $M_{j}$ (resp. of $F$ to $M_{j}$ ). Let

$$
\mathcal{H}_{j}^{k}=\operatorname{ker} A_{j}^{k}, \quad j=1,2 .
$$

By the main result from [4], for $k \geq k_{0}$ (for some positive integer $k_{0}$ ) the space $\mathcal{H}_{j}^{k}$ consists entirely of even elements with respect to the natural grading of the Spin $^{c}$ Clifford bundle over a symplectic manifold, and therefore its dimension is the index of the Dirac operator $A_{j}^{k}$. For $k \geq k_{0}$ the space $\mathcal{H}_{j}^{k}$ is called the $\operatorname{Spin}^{c}$ quantization of $M_{j}$.

To simplify the notation, we will occasionally omit the superscript $k$.

Cauchy data spaces. The $L^{2}$ space of the bundle $E \otimes L^{k} \rightarrow \partial X$ is naturally a symplectic vector space with symplectic form $\Omega$ given by

$$
\Omega\left(\psi_{1}, \psi_{2}\right):=\int_{\partial X}\left\langle\left\langle\mathcal{J} \psi_{1}, \psi_{2}\right\rangle\right\rangle_{x} d V(x),
$$

where $\mathcal{J}$ denotes the continuous $L^{2}$ extension of $c(\eta)$ on the boundary, and $\langle\langle\cdot, \cdot\rangle\rangle$ the fiberwise inner product.

We will denote by $\gamma$ the operator restricting sections of $F \otimes \mathcal{L}^{k}$ to the boundary, and by $W^{s}\left(F \otimes \mathcal{L}^{k}\right)$ the space of $s$-Sobolev sections of $F \otimes \mathcal{L}^{k}$. The operator $\gamma: W^{s}\left(F \otimes \mathcal{L}^{k}\right) \rightarrow W^{s-1 / 2}\left(E \otimes L^{k}\right)$ is continuous for $s>1 / 2$, and remains continuous for $s=1 / 2$ into $L^{2}\left(E \otimes L^{k}\right)$ with domain restricted to

$$
\mathcal{K}_{1 / 2}^{k}=\left\{\psi \in C^{\infty}\left(F \otimes \mathcal{L}^{k}\right) \mid D^{k} \psi=0 \text { in } X\right\} \cap W^{1 / 2}\left(F \otimes \mathcal{L}^{k}\right) .
$$

(See [7].) The space

$$
\Lambda\left(D^{k}\right)=\left\{\gamma \psi \mid \psi \in \mathcal{K}_{1 / 2}^{k}\right\}
$$


is called the Cauchy data space of the Dirac operator $D^{k}$. For us the most important property of this space is given in the following lemma, which is a direct consequence of Prop. 3.2 of [8].

Lemma 2.1. The Cauchy data space $\Lambda(D) \subset L^{2}\left(\partial X, E \otimes L^{k}\right)$ is a Lagrangian subspace with respect to the symplectic structure defined in (2.8).

We let $\mathcal{H}_{\geq} \subset L^{2}\left(\partial X, E \otimes L^{k}\right)$ be the space spanned by the eigenvectors of $A^{k}$ corresponding to non-negative eigenvalues.

Lemma 2.2 (Cf. [16].). The space $\mathcal{H}_{>}$is coisotropic in $L^{2}\left(\partial X, E \otimes L^{k}\right)$ and its symplectic orthogonal is the space $\hat{\mathcal{H}}_{>}$spanned by the eigenvectors of $A^{k}$ corresponding to positive eigenvalues.

It follows that the reduction $\mathcal{H}_{\geq} / \mathcal{H}_{>}$can be naturally identified with

$$
\mathcal{H}_{0}^{k}=\operatorname{ker} A^{k}
$$

and the projection onto the reduced space with the orthogonal projection $\pi: \mathcal{H}_{\geq} \rightarrow \mathcal{H}_{0}$. Of central importance to us is the reduction of the Cauchy data space

$$
L(D):=\pi\left(\mathcal{H}_{\geq} \cap \Lambda(D)\right),
$$

which is a Lagrangian subspace of the kernel of $A^{k}$. Explicitly, $L(D)$ consists of those elements in ker $A^{k}$ that are the $\mathcal{H}_{0}$ components of the Cauchy data in $\mathcal{H}_{\geq}$.

The Definition. Notice that $\mathcal{H}_{0}^{k}=\operatorname{ker} A^{k}$ is the direct sum

$$
\mathcal{H}_{0}^{k}=\mathcal{H}_{1}^{k} \oplus \mathcal{H}_{2}^{k},
$$

where $\mathcal{H}_{j}$ consists of those elements of $\operatorname{ker} A^{k}$ which are supported on $M_{j}$. We further have the decomposition according to the eigenspaces of $\mathcal{J}$ :

$$
\mathcal{H}_{j}^{k}=\mathcal{H}_{j}^{k+} \oplus \mathcal{H}_{j}^{k-} \quad j=1,2 .
$$

Since the boundary orientation of $M_{2}$ is the opposite of its symplectic orientation, in the above decomposition $\mathcal{H}_{2}^{+}$resp. $\mathcal{H}_{2}^{-}$consists of the odd resp. even elements of $\mathcal{H}_{2}$. Formally (for $k=1$ ), the quantization of $M_{1}$ is the virtual vector space $\mathcal{H}_{1}^{+}-\mathcal{H}_{1}^{-}$, while that of $M_{2}$ is $\mathcal{H}_{2}^{-}-\mathcal{H}_{2}^{+}$. Let $U^{k}: \mathcal{H}_{1}^{k} \oplus \mathcal{H}_{2}^{k} \rightarrow \mathcal{H}_{1}^{k} \oplus \mathcal{H}_{2}^{k}$ be the orthogonal reflection across the subspace $L(D) . U^{k}$ can be thought of as a morphism between virtual vector spaces:

Proposition 2.3. (Cf. [15].) The operator $U^{k}$ is unitary and anti-commutes with $\mathcal{J}$. It therefore restricts to a unitary operator $\mathcal{U}^{k}: \mathcal{H}_{1}^{k+} \rightarrow \mathcal{H}_{2}^{k-}$ (and also to a unitary operator $\left.\mathcal{H}_{1}^{k-} \rightarrow \mathcal{H}_{2}^{k+}\right)$.

The anti-commutativity of $U^{k}$ and $\mathcal{J}$ is equivalent to $L(D)$ being Lagrangian. Note also that for $k \geq k_{0}$ the $\operatorname{Spin}^{c}$ quantization of $M_{1}$ (resp. $M_{2}$ ) is precisely $\mathcal{H}_{1}^{k+}$ (resp. $\mathcal{H}_{2}^{k-}$ ).

We can finally state our definition: 
Definition 2.4. The quantization of $(X, \sigma)$ associated to the choice of compatible metric is the sequence of unitary operators $\mathcal{U}^{k}: \mathcal{H}_{1}^{k} \rightarrow \mathcal{H}_{2}^{k},\left(k \geq k_{0}\right)$.

\section{Semiclassical properties of the quantized cobordism}

We now describe the semiclassical (i.e. large $k$ ) behavior of the operator $\mathcal{U}^{k}$. To make sense out of this, we consider the pull-back of the bundle $E_{j}(j=1,2)$ to the unit circle bundle $P_{j} \rightarrow M_{j}$ of $L_{j}^{*} \rightarrow M_{j}$ :

$$
\mathcal{E}_{j}:=\pi^{*} E_{j}, \quad \pi: P_{j} \rightarrow M_{j} .
$$

The circle group acts on $\mathcal{E}_{j}$, and in the isotypical decomposition

$$
L^{2}\left(P_{j}, \mathcal{E}_{j}\right)=\bigoplus_{k \in \mathbb{Z}} L^{2}\left(P_{j}, \mathcal{E}_{j}\right)_{k}
$$

the space $L^{2}\left(P_{j}, \mathcal{E}_{j}\right)_{k}$ is naturally isomorphic to $L^{2}\left(M_{j}, E_{j} \otimes L_{j}^{k}\right)$. Under this isomorphism the family of Dirac operators $\left\{A_{j}^{k}\right\}$ is induced by a single firstorder operator $\mathcal{A}_{j}$, acting on $C^{\infty}\left(P_{j}, \mathcal{E}_{j}\right)$, which is transversally elliptic to the $S^{1}$ action and commutes with it. If we set $\mathcal{Q}_{j}=\operatorname{ker} \mathcal{A}_{j} \cap \bigoplus_{k \geq k_{0}} L^{2}\left(P_{j}, \mathcal{E}_{j}\right)_{k}$, then the quantization $\mathcal{H}_{j}^{k}$ is the space of $k^{t h}$ isotypes in $\mathcal{Q}_{j}$, and the projector $\Pi_{j}^{k}$ is the $k^{t h}$ Fourier component of the orthogonal projector $\Pi_{j}$ onto $\mathcal{Q}_{j}$. The family $\left\{\mathcal{U}^{k}\right\}$ (the quantized cobordism) also lifts to a single operator $\mathcal{U}: \mathcal{Q}_{1} \rightarrow \mathcal{Q}_{2}$, and the large $k$ behavior of $\mathcal{U}^{k}$ can be understood in terms of a precise description of the microlocal structure of $\mathcal{U}$.

Before stating the main theorem on the structure of $\mathcal{U}$, we make some further comments. The connection form, $\alpha$, of the circle bundle $\pi: P \rightarrow M$ satisfies $d \alpha=\pi^{*} \omega, \iota_{\partial_{\theta}} \alpha=1$, where $\partial_{\theta}$ is the infinitesimal generator of the $S^{1}$ action. As usual, the connection induces bundle splittings

$$
T P=H \oplus V, \quad T^{*} P=H^{*} \oplus V^{*}
$$

where $H$ is the kernel of $\alpha, V$ is spanned by $\partial_{\theta}$ and $V^{*}$ is spanned by $\alpha$. We put the unique $S^{1}$-invariant metric on $P$ that makes $\pi$ a Riemannian submersion with totally geodesic fibers (of length $2 \pi$ ). We will denote by

$$
\begin{array}{rlc}
\tau: T^{*} P & \rightarrow & \mathbb{R} \\
(p, \zeta) & \mapsto\left\langle\zeta, \partial_{\theta}\right\rangle
\end{array}
$$

the symbol of $D_{\theta}:=\frac{1}{i} \partial_{\theta}$. Notice that, for every $\zeta \in T_{p}^{*} P, \tau(\zeta) \alpha_{p}$ is the $V^{*}$ component of $\zeta$ in its $H^{*} \oplus V^{*}$ decomposition.

Recall that the square of Dirac operator $A^{k}$ is a (generalized) Laplacian on the bundle $E \otimes L^{k} \rightarrow M$, with scalar principal symbol $h(x, \xi)=|\xi|^{2}$. The square of $\mathcal{A}_{j}$ is therefore a "horizontal" Laplacian, $\square_{j}$, on the bundle $\mathcal{E}_{j}$, with scalar principal symbol $\tilde{h}(z, \zeta)=\left|\zeta_{h o r}\right|^{2}$, where $\zeta_{\text {hor }}$ is the horizontal component of $\zeta$. It follows that the characteristic set of $\square_{j}$ is

$$
\Sigma_{j}=V^{*} \backslash\{0\} .
$$


Since $A^{k}$ is self-adjoint, we have $\operatorname{ker} A^{k}=\operatorname{ker}\left(A^{k}\right)^{2}$ and therefore $\operatorname{ker} \mathcal{A}_{j}=$ ker $\square_{j}$. It is also well known that any distribution in the span of spaces $L^{2}(P, \mathcal{E})_{k}$, $k>0$ has wave front set contained in $\{\tau \geq 0\}$ hence for all $\psi \in \mathcal{Q}_{j}$, the wavefront set of $\psi$ is contained in $\Sigma_{j}^{+}:=\Sigma_{j} \cap\{\tau \geq 0\}$.

The main theorem. Recall that $\nu$ is the vector field on $X$ spanning the kernel of $\sigma$. We denote by $\bar{\nu}$ its horizontal lift to the circle bundle $Z \rightarrow X$ of $\mathcal{L}^{*} \rightarrow X$, and let $H(z, \zeta)=\left\langle\bar{\nu}_{z}, \zeta\right\rangle$ be the symbol of $\bar{\nu}$. The Hamilton vector field of $H$ (on $T^{*} Z$ ) will be denoted by $\Xi_{H}$. Let $P_{j}^{\circ}=\pi_{j}^{-1}\left(M_{j}^{\circ}\right)$ denote the set of points of $P_{j}$ projecting onto $M_{j}^{\circ}$ (the complement of the surgery set). Notice that the boundary of $Z$ is $P_{1} \amalg P_{2}$, and that one can naturally embed $T^{*} P_{j}$ into $T^{*} Z$ as the annihilator of the span of $\bar{\nu}$ restricted to $P_{j}$. We next define the isotropic submanifold $W \subset T^{*}\left(P_{2}^{\circ} \times P_{1}^{\circ}\right)$ :

$$
\begin{aligned}
W:=\left\{\left(p_{2}, \xi_{2} ; p_{1}, \xi_{1}\right) \mid\left(p_{2}, \xi_{2}\right) \in\right. & \Sigma_{2}^{+} \text {and }\left(p_{1}, \xi_{1}\right) \in \Sigma_{1}^{+} \\
& \text {are connected by a flow line of } \left.\Xi_{H}\right\} .
\end{aligned}
$$

As is customary we let $W^{\prime}:=\left\{\left(p_{1}, \xi_{1} ; p_{2},-\xi_{2}\right) \mid\left(p_{1}, \xi_{1} ; p_{2}, \xi_{2}\right) \in W\right\}$. We can now state our main theorem on the microlocal structure of the quantized cobordism map, $\mathcal{U}$. Let $\mathcal{U}(z, x)$ be the Schwartz kernel of $\mathcal{U}: \mathcal{Q}_{1} \rightarrow \mathcal{Q}_{2}$, which is a distributional section of the bundle $\mathcal{E}_{2} \otimes \mathcal{E}_{1}^{*} \rightarrow P_{2} \times P_{1}$. (We define $\mathcal{U}$ to be zero on the orthogonal complement of $\mathcal{Q}_{1}$.)

Theorem 3.1. (L. Korpás, [13].) Let $\tilde{\mathcal{U}}(z, x)$ be the restriction of $\mathcal{U}(z, x)$ to the manifold $P_{2}^{\circ} \times P_{1}^{\circ}$. Then $\tilde{\mathcal{U}}(z, x)$ is an Hermite-Fourier distribution (in the sense of Boutet de Monvel and Guillemin [6]) in the space $I^{1 / 2}\left(P_{2}^{\circ} \times P_{1}^{\circ}, \mathcal{E}_{2} \otimes\right.$ $\left.\mathcal{E}_{1}^{*} ; W^{\prime}\right)$. In particular, in the semiclassical regime, the operator $\mathcal{U}^{k}$ propagates singularities along the relation $\Gamma$, given in (1.2).

For a description of the symbol of the operator $\mathcal{U}$ we refer to [13].

The proof of this theorem involves several steps which are of independent interest. We now describe two of them.

The family of Dirac operators $\left\{D^{k}\right\}$ is also generated by a single operator $\mathcal{D}$ on the bundle $\mathcal{F}:=\pi^{*} F \rightarrow Z$. The idea of the proof is then to realize $\mathcal{U}$ as the composition of the fundamental solution of a boundary value problem for $\mathcal{D}$ and the projector $\Pi$, which we will call the $\operatorname{Spin}^{c}$ Szegö projector.

Theorem 3.2. The Spin ${ }^{c}$ Szegö projector $\Pi$ is an Hermite operator of order zero in the sense of [6], i.e. it has a distribution kernel which is a HermiteFourier distribution in the class $I^{1 / 2}\left(P \times P, \mathcal{E} \otimes \mathcal{E}^{*} ; \Sigma^{+\sharp}\right)$, where

$$
\Sigma^{+\sharp}=\left\{(x, \xi ; x,-\xi) \mid(x, \xi) \in \Sigma^{+}\right\} \subset T^{*}(P \times P) .
$$

and $\Sigma^{+}$was defined previously. 
The boundary problem involved in the proof of Theorem 3.1 is given as follows:

$$
\left\{\begin{array}{l}
\mathcal{D} \psi=0 \\
\left.\psi\right|_{P_{1}}=\phi,
\end{array}\right.
$$

with $\phi \in \mathcal{Q}_{1} \cap \mathcal{E}^{\prime}\left(P_{1}^{\circ}\right)$, a distribution in $\mathcal{Q}_{1}$, compactly supported away from the "surgery set" set $P_{1} \backslash P_{1}^{\circ}$.

Theorem 3.3. The fundamental solution of this boundary problem is an Hermite-Fourier distribution in $I^{\frac{1}{2}}\left(Z^{\circ} \times P_{1}^{\circ}, \mathcal{F} \otimes \mathcal{E}^{*}, Y\right)$, where $Z^{\circ} \subset Z$ is the set reachable from $P_{1}^{\circ}$ along the flow of $\bar{\nu}$ and $Y$ is the flow-relation defined by $\Xi_{H}$ on $T^{*} Z^{\circ} \times T^{*} P_{1}^{\circ}$, analogously to $W$.

This solution can be constructed explicitly using the symbol calculus of Hermite-Fourier distributions [13].

\section{Examples}

In this final section we describe some examples of cobordisms between surfaces. By work of V. Ginzburg [11], the cobordism invariants of symplectic surfaces are their Euler characteristics and symplectic volumes. Here we consider Riemann surfaces with a fixed complex structure, but with the symplectic form and the metric rescaled by a factor of $1 / 2$ when $g \geq 2$.

Theta functions and holomorphic differentials. Consider integral symplectic manifolds $M_{1}=\Sigma_{2} \coprod S$ and $M_{2}=T \coprod T$, where $T$ is a torus, $S$ is the Riemann sphere and $\Sigma_{2}$ is a genus 2 surface. (See Figure 1.) Since their Euler characteristics and symplectic volumes are equal, they are cobordant: $M_{1} \coprod M_{2}^{-}=$ $\partial X$ for some symplectic cobordism $X$. (For an explicit construction of such cobordisms see [12].) We let $L_{0}$ denote the fundamental prequantum line bundle, i.e. the one corresponding to the positive generator of the second cohomology group of the surface. Then, in this example, our operator $\mathcal{U}^{k}$ is an isomorphism from the space of theta functions $H^{0}\left(T, \mathcal{O}\left(L_{0}^{k}\right)\right) \oplus H^{0}\left(T, \mathcal{O}\left(L_{0}^{k}\right)\right)$ to the direct sum of the holomorphic differentials $H^{0}\left(\Sigma_{2}, \mathcal{O}\left(L_{0}^{k}\right)\right)$ and the space of polynomials of degree $k$ in one complex variable.

The quantized "baker's transformation." Symplectic cobordisms can be used to realize certain piecewise-smooth area preserving transformations of a symplectic manifold, and therefore our constructions can be used to quantize such maps. For example, let $M=T$ be the standard two torus. The baker's map is the following discontinuous map: $B:[0,1) \times[0,1) \rightarrow[0,1) \times[0,1)$ :

$$
B(x, y)= \begin{cases}\left(2 x, \frac{y}{2}\right), & \text { if } \quad 0 \leq x<\frac{1}{2} \\ \left(2 x-1, \frac{y+1}{2}\right), & \text { if } \quad \frac{1}{2} \leq x<1 .\end{cases}
$$

We will think of $B$ as operating on the torus obtained by the usual identifications of $[0,1] \times[0,1]$. In the following paragraphs we briefly describe how to construct 


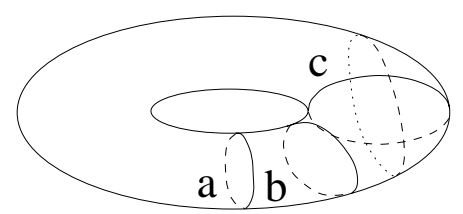

$\mathrm{T}$

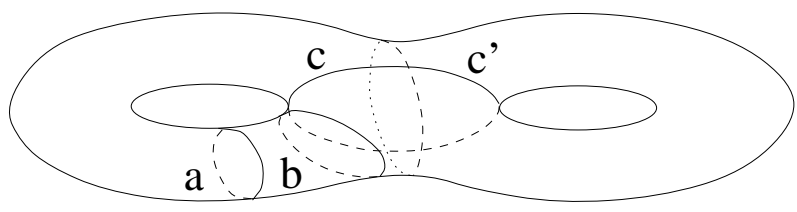

$\Sigma_{2}$



$\mathrm{T}$

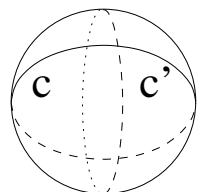

$\mathrm{S}$

FiguRE 1. Surgery of two tori via cobordism. (Dotted-dashed lines show the locations of the cuts.) The foliation of the tori by the curves $a, b, c, c^{\prime}$ etc. is mapped to a singular foliation of the genus 2 surface and the sphere. Note that some leaves of the foliation get cut and pasted.

an integral symplectic cobordism, $X$, from $T$ to itself whose characteristic relation (1.2) is precisely the smooth part of the graph of $B$. It would be interesting to compare the quantization of this symplectic cobordism with the definition of the quantized baker's map that Balazs and Voros gave in [2], or with the recent construction of Rubin and Salwen [17]. We believe that our definition agrees with theirs at least to leading order as $k \rightarrow \infty$.

To construct a cobordism realizing the baker's transformation, we first describe a cobordism between a torus of volume 2 and two tori, each of volume 1 . (This construction can also be found in [12].) Let $P$ be a "pair of pants" surface with volume form $\Omega$ and set $Z=P \times S^{1}$. Let $\theta$ denote the canonical local coordinate on the cirle, then $\Omega \wedge d \theta$ is a volume form on $Z$. Choose a divergence-free vector field $v$ on $P$ which is transverse to the boundary and vanishes exactly at one point, $p$, as shown on Fig. 2. We set $\nu=v+\chi \partial_{\theta}$, where $\chi$ is a smooth cutoff function on $P$, zero outside of a small neighborhood of the point $p$, and $\chi(p)=1$. Then $\sigma=\imath_{\nu}(\Omega \wedge d \theta)$ will give $X$ the structure of a symplectic cobordism. With appropriate choice of the vector field $v$ and the volume form $\Omega$ we can achieve that the volumes of the boundary tori are 2 and 1 , as required.

Note that, although the area is preserved, the flow will introduce an "infinite twisting" since the vector field $v$ on the pair of pants vanishes at the point $p$. (See Fig. 3a for an illustration. On the figure horizontal lines indicate a foliation of half of the initial torus parallel to the boundary circle of $P$.) The twisting can be "undone" if we modify the original vector field on $P$ as follows. Define the divergence-free vector field on the cylinder $I \times S^{1}$ as shown on Fig. 3b. (This 




Figure 2. A divergence-free vector field on a pair of pants which vanishes at one point.


Figure 3. (a) "Twisting" of a foliation of the torus under the flow of $\nu$. (b) Part of a construction to "untwist" the foliation.

vector field can be chosen Hamiltonian.) Now attach this cylinder along the boundary circle to each of the boundary circles $S_{1}^{P}$ and $S_{2}^{P}$ of $P$, and construct the 2-form $\sigma$ as before, by choosing the appropriate cutoff functions, centered at $A, B$ and $p$. We let $X$ denote the cobordism between one torus and the disjoint union of two tori, modified by this procedure.

Finally, let $X^{-}$denote the manifold $X$ with the vector field $\nu$ oppositely oriented. The cobordism realizing the baker's map can be obtained by gluing $X$ and $X^{-}$along the two smaller tori, as follows. Near the boundary $X$ has the form:

$$
(-1,0] \times S^{1} \times S_{1}^{P} \coprod(-1,0] \times S^{1} \times S_{2}^{P} .
$$

To obtain the cobordism, we identify the respective circles in the boundary tori:

$$
S^{1} \times S_{1}^{P} \quad \text { with } \quad S_{1}^{P^{-}} \times S^{1}
$$

and

$$
S^{1} \times S_{2}^{P} \quad \text { with } \quad S_{2}^{P^{-}} \times S^{1} .
$$

$\left(P^{-}\right.$denotes the pair of pants with the vector field $v$ replaced by $-v$.) It is easy to check that the flow of the vector field indeed realizes the baker's map.

\section{Acknowledgments}

We wish to thank Liviu Nicolaescu for many useful discussions. 


\section{References}

[1] V.I. Arnold, Mathematical Methods of Classical Mechanics, (K. Vogtmann and A. Weinstein, trans.), Graduate Texts in Mathematics, 60., Springer-Verlag, New York-Heidelberg, 1978.

[2] N.L. Balazs and A. Voros, The quantized baker's transformation, Ann. Physics 190 (1989), $1-31$.

[3] N. Berline, E. Getzler, and M. Vergne, Heat kernels and Dirac operators, Springer-Verlag, Berlin-Heidelberg, 1992.

[4] D. Borthwick and A. Uribe, Almost complex structures and geometric quantization, Math. Res. Lett. 3 (1996), 845-861.

[5] L. Boutet de Monvel, Hypoelliptic operators with double characteristics and related pseudodifferential operators, Comm. Pure Appl. Math. 27 (1974), 585-639.

[6] L. Boutet de Monvel and V. Guillemin, The spectral theory of Toeplitz operators, Princeton University Press, Princeton, N.J., 1981.

[7] B. Booss-Bavnbek and K. Wojciechowski, Elliptic boundary problems for Dirac operators, Birkhäuser Boston, Inc., Boston, MA, 1993.

[8] B. Booss and K. Wojciechowski, Desuspension of splitting elliptic symbols II, Ann. Glob. Anal. Geom. 4 (1986), 349-400.

[9] J.J. Duistermaat, The Heat Kernel Lefschetz Fixed Point Formula for the Spin-c Dirac Operator, Progress in Nonlinear Differential Equations and their Applications, 18., Birkhäuser Boston, Inc., Boston, MA, 1996.

[10] V. Ginzburg, V. Guillemin and Y. Karshon, Cobordism theory and localization formulas for Hamiltonian group actions, Internat. Math. Res. Notices, (1996), 221-234.

[11] V.L. Ginzburg, Calculation of contact and symplectic cobordism groups, Topology 31 (1992), 767-773.

[12] V.L. Ginzburg, Cobordisms of symplectic and contact manifolds, Funkt. Anal. i Ego Prilozhen. 23 (1987), 27-31. (Translated from the Russian.)

[13] L. Korpás, Ph.D. Thesis, in preparation.

[14] H.B. Lawson, Jr. and M.-L. Michelsohn, Spin geometry, Princeton University Press, 1989.

[15] L. Nicolaescu, On the cobordism invariance of the index of Dirac operators, Proc. Amer. Math. Soc. 125 (1997), 2797-2801.

[16] L. Nicolaescu, The Maslov index, the spectral flow, and decompositions of manifolds, Duke Math. J. 80 (1995), 485-533.

[17] R. Rubin and N. Salwen, A canonical quantization of the Baker's map, Ann. Physics 269 (1998), 159-181.

[18] J.-M. Souriau, Structure des systèmes dynamiques, Dunod, Paris 1970.

Department of Mathematics, University of Michigan, Ann Arbor, Mi 48109

E-mail address: korpas@math.lsa.umich.edu

Department of Mathematics, University of Michigan, Ann Arbor, Mi 48109

E-mail address: uribe@math.lsa.umich.edu 